\title{
VISIONES DE LOS ESTUDIANTES DE LA DOCENCIA UNIVERSITARIA MEDIADA POR TIC
}

\section{VISIONS OF STUDENTS AT THE UNIVERSITY TEACHING ICT MEDIATED}

\author{
Dra. Ma José Rubio Hurtado ${ }^{1}$ \\ mjrubio@ub.edu \\ Dra. Anna Escofet Roig² \\ anna.escofet@ub.edu
}

\author{
${ }^{(1)}$ Universidad de Barcelona. Facultad de Pedagogía. \\ Departamento de Métodos de Investigación y Diagnóstico en Educación. \\ Passeig de la Vall d'Hebron, 171, 08035, Barcelona (España) \\ (2) Universidad de Barcelona. Facultad de Pedagogía. \\ Departamento de Teoría e Historia de la Educación. \\ Passeig de la Vall d'Hebron 171, 08035, Barcelona (España)
}

El objetivo de la investigación fue conocer la percepción del alumnado universitario en relación a los aspectos que explican la experiencia educativa con TIC en las tres dimensiones consideradas por Garrison, Anderson y Archer (2000): didáctica, cognitiva y social. La muestra la integraron 317 estudiantes de carreras de Medicina y Educación de la Universidad de Barcelona, a los cuales se les aplicó una escala tipo Likert diseñada específicamente para el estudio. Los resultados, coincidentes con los de otras investigaciones, muestran índices más elevados de presencia cognitiva y didáctica, frente a la presencia social, existiendo diferencias significativas en función de los estudios.

Palabras clave: TIC, educación superior, aprendizaje, experiencia educativa.

The aim of the research was to understand the perception of university students in relation to the aspects that explain the educational experience with ICT in the three dimensions considered by Garrison, Anderson and Archer (2000): didactic, cognitive and social. The sample was composed of 317 students pursuing careers in Medicine and Education, University of Barcelona, to which was applied a Likert scale designed specifically for the study. The results, which are consistent with those of other studies, show higher rates of cognitive and teaching than social presence, and significant differences are found depending on the student career.

Keywords: ICT, higher education, learning, educational experience. 


\section{Introducción.}

La introducción de las tecnologías de la información y la comunicación en las aulas universitarias ha sido crucial en la docencia universitaria y el aprendizaje. Varios estudios (Fraser, 2002; New Media Consortium, 2010) ponen de manifiesto las posibilidades que ofrecen las TIC y el punto de inflexión que representan para los entornos de aprendizaje tradicionales, dando lugar a la formación virtual y el blended learning. En el caso del aprendizaje virtual, nos estamos refiriendo a los ambientes de aprendizaje mediados por plataformas tecnológicas (Harasim, 1990; McIsaac \& Gunawardena, 1996) y, en el caso del blended learning, nos estamos refiriendo a un ambiente de aprendizaje que combinan la enseñanza presencial con el uso de las TIC (Bersin, 2004; Thorne, 2003; Ardizzone \& Rivoltella, 2003).

En este marco, Garrison, Anderson y Archer (2000) proponen la creación de comunidades de indagación, cuyo establecimiento favorece la realización de la experiencia educativa. En estas comunidades, el aprendizaje tiene lugar por la interacción de tres componentes principales: la presencia cognitiva, la presencia didáctica y la presencia social. La presencia cognitiva, definida por los autores, como el grado en que los participantes en cualquier tipo de comunidad de indagación son capaces de construir significado mediante la comunicación establecida. La presencia didáctica, que incluye el diseño y el desarrollo de las secuencias de aprendizaje, proporcionando los contenidos de la materia y facilitando el estudio activo. Ello incluye la selección, la organización, y la presentación inicial de los contenidos, así como el diseño y el desarrollo de las actividades de aprendizaje y evaluación. Finalmente, la presencia social es definida como la capacidad de los estudiantes de proyectarse socialmente y emocionalmente en una comunidad de indagación. La importancia de este elemento es su función como apoyo a la presencia cognitiva, facilitando indirectamente el proceso de pensamiento crítico. La presencia social apoya a los objetivos cognitivos por su capacidad de promover el pensamiento crítico en una comunidad de estudiantes. En la intersección de estos tres elementos se crea un contexto estimulante, que facilita el discurso crítico y la reflexión para la construcción de significado y conocimiento, y que da sentido a una comunidad con objetivos educativos consiguiendo una adecuada experiencia de aprendizaje. Más allá de la instrucción directa, el modelo también centra la atención en el diseño y la organización de la enseñanza teniendo en cuenta la necesidad de los estudiantes de sentir que están participando en una comunidad social (Rourke, Anderson, Garrison \& Archer, 1999; Shea \& Bidjerano, 2009).

Una parte importante y creciente de la literatura ha tratado de articular y ampliar la comunidad de indagación. Así, se incluyen los intentos de esbozar y validar los aspectos particulares del modelo (Arbaugh, 2007; Garrison, 2007; Garrison \& Cleveland-Innes, 2005; Shea, Li, Swan \& Pickett, 2005). Estudios recientes analizan la interdependencia de sus diferentes componentes, como el de Shea y Bidjerano (2009) que estudia el impacto de la presencia docente en el desarrollo de la presencia social y el de Shea, Li y Pickett (2006) que investiga la adecuación del marco para describir y explicar las diferencias en los resultados del aprendizaje en entornos semipresenciales y totalmente en línea. Cabe señalar que el marco teórico sugiere que los componentes no existen en forma aislada (Garrison, Anderson \& Archer, 2000), sino que 
más bien cada uno puede ser visto como la superposición en su conjunto. Esta representación implica que cada una de las formas de presencia se relaciona con las otras, y que las tres se combinan dentro de la comunidad de indagación, aunque quedan por confirmar las relaciones y la influencia entre las mismas (Shea, Li \& Pickett, 2006). Por último, Coll, Bustos y Engel (2011) analizan el rol que tanto profesores como alumnos toman de manera activa en la presencia docente en las aulas universitarias. Para estos autores, la presencia docente no es patrimonio exclusivo del profesor, sino que se distribuye en grados y modalidades diversas entre los participantes.

\section{Objetivos.}

El objetivo principal de la investigación fue conocer la percepción del alumnado universitario en relación a los aspectos que explican la experiencia educativa con TIC en las tres dimensiones mencionadas. Como segundo objetivo se pretendió explorar posibles relaciones entre las tres dimensiones consideradas y variables sociodemográficas como el sexo, los estudios, el curso, y la frecuencia del uso de TIC.

\section{Metodología.}

El método de estudio fue la encuesta, método apropiado cuando el objetivo es conocer opiniones, creencias o actitudes de un conjunto de personas (Buendía, Colás \& Hernández, 1998).

La muestra de participantes en la investigación estuvo formada por estudiantes pertenecientes a tres titulaciones de la Universidad de Barcelona: Estudios de Medicina, Estudios de Educación Social y Estudios de Maestro durante el curso 2010-
2011. El tamaño de la muestra fue de 317 estudiantes para un nivel de confianza del $95 \%$ y un error del 5,6\% para una población de 47.000 estudiantes matriculados en la Universidad de Barcelona. El tamaño se consideró razonablemente adecuado para el estudio según la recomendación de Kass y Tinsley (1979) de tomar ente 5 y 10 participantes por ítem del instrumento de recogida datos (en nuestro caso un instrumento de 36 ítems). Para la selección de los participantes se utilizó un muestreo accidental (Hernández Sampieri, Fernández Collado \& Baptista Lucio, 2006), caracterizado por la selección de casos de fácil acceso.

El instrumento de recogida de datos fue un cuestionario formado por 36 ítems tipo escala Likert de cinco grados (muy en desacuerdo 1-, en desacuerdo -2-, ni de acuerdo ni en desacuerdo -3-, de acuerdo -4-, muy de acuerdo -5-) orientado a conocer la percepción del alumnado en las dimensiones didáctica, cognitiva y social conceptualizadas por Garrison, Anderson y Archer (2000), las cuales explican la experiencia educativa con TIC. Para caracterizar la muestra el cuestionario contenía preguntas de tipo sociodemográfico como sexo del alumnado, estudios en los que se encuentra, curso más alto del que está matriculado, y frecuencia de uso que hace de diferentes tipologías de TIC.

Para crear la escala se identificaron previamente un conjunto de indicadores para cada una de las tres dimensiones propuestas por Garrison, Anderson y Archer (2000):

1. Los indicadores para la dimensión presencia cognitiva incluyen provocar un acontecimiento (reconocer el problema, proponer una pregunta); fomentar la exploración (intercambiar información, discutir ambigüedades); promover la integración (conectar ideas, crear soluciones); conseguir la resolución (aplicar 
FACTOR 1

\begin{tabular}{|c|c|}
\hline FACTOR 1 & $\begin{array}{c}\text { PORCENTAJE DE } \\
\text { VARIANZA EXPLICADA }\end{array}$ \\
\hline Presencia cognitiva & $23.57 \%$ \\
\hline Ítems & Saturación \\
\hline 1. Las TIC me ayudan a hacer más rapidamente las tareas universitarias & .721 \\
\hline 2. Considero que las TIC son importantes en mis aprendizajes & .705 \\
\hline 3. Con las TIC aprobar las asignaturas es más fácil & 673 \\
\hline 4. Las TIC me ayudan a hacer mejor las tareas universitarias & .667 \\
\hline 5. Las TIC me facilitan el seguimiento de la asignatura & 657 \\
\hline 6. Las TIC me permiten asociar ideas & .543 \\
\hline 7. Las TIC me permiten aplicar nuevas id eas & .521 \\
\hline 8. Sin las TIC me resultaría difícil hacer las tareas universitarias & .498 \\
\hline 9. Las TIC me ayudan a adquirir conocimientos relacionados con la asignatura & .497 \\
\hline 10. Las TIC me permiten aplicar el conocimiento adquirido & .480 \\
\hline 11. Cuando quiero saber más sobre algún tema, utilizo las TIC & .452 \\
\hline 12. Las TIC permiten establecer un calendario de trabajo & .451 \\
\hline 13. Las TIC facilitan los procesos de autoevaluación & .400 \\
\hline FACTOR 2 & $\begin{array}{c}\text { PORCENTAJE DE } \\
\text { VARIANZA EXPLICADA }\end{array}$ \\
\hline Presencia didáctica & $36.58 \%$ \\
\hline Ítems & Saturación \\
\hline 14. Las TIC me facilitan recibir ayuda del profesor/a & .654 \\
\hline 15. Las TIC facilitan la resolución de mis dudas & .621 \\
\hline 16. Las TIC dinamizan las contribuciones de los compañeros/as & .586 \\
\hline 17. Con las TIC resuelvo mejor las dudas & .570 \\
\hline 18. Las TIC me facilitan el diagnóstico en mis errores de aprend izaje & .549 \\
\hline 19. Las TIC me permiten valorar mis avances en la asignatura & .508 \\
\hline 20. Las TIC me facilitan la integración de conocimientos de diferentes fuentes & .465 \\
\hline
\end{tabular}

Tabla 1. Componentes del análisis factorial de la escala.

PORCENTAJE DE VARIANZA EXPLICADA

$23.57 \%$

Saración

.721 
FACTOR 2

PORCENTAJE DE VARIANZA EXPLICADA

\begin{tabular}{|lr}
\hline Presencia didáctica & $\mathbf{3 6 . 5 8 \%}$ \\
\hline Ítems & Sa turación \\
\hline 21. Las TIC me permiten confirmar lo que he aprendido & .489 \\
22. El debate con los compañeros/as se ve potenciado con el uso de las TIC & .488 \\
23. Las TIC me permiten evaluar mis procesos de aprendizaje & .475 \\
24. Las TIC facilitan la presentación de los contenidos & .446 \\
25. Las TIC facilitan pautar la metodología de trabajo & .428
\end{tabular}

FACTOR 3

PORCENTAJE DE VARIANZA EXPLICADA

$\begin{array}{lr}\text { Presencia social } & \mathbf{5 0 . 3 1 \%}\end{array}$

Ítems

Sa turación

26. Las TIC me facilitan que pueda expresarme abiertamente

27. Las TIC facilitan las expresiones de aprecio con el grupo 797

28. Las TIC facilitan el establecimiento de un clima agradable en el aula 776

29. Las TIC me facilitan mostrarme tal y como soy

30. Con las TIC expreso más libremente mis emociones $\quad .723$

31. Las TIC me permiten crear red con mis compañeros/as $\quad .653$

32. Las TIC me permiten proyectar públicamente lo que hago en las asignaturas $\quad .600$

33. Las TIC me facilitan hacer preguntas a los demás

34. Explico mis problemas (personales, de clase...) al profesor/a por medio de las TIC

35. Las TIC facilitan que el profesor/a puede dedicarse más al grupo

36. Las TIC me permiten una mejor comunicación con mis compañeros/as

Tabla 1. Componentes del análisis factorial de la escala.

nuevas ideas, evaluar de manera crítica soluciones).

2. Los indicadores para la dimensión presencia didáctica incluyen dirección de instrucción (estructuración del contenido, propuesta de temas de discusión, establecimiento de grupos de discusión); construcción de la comprensión (compartir valores y significados personales, expresar el acuerdo, buscar el acuerdo general); e instrucción directa (focalizar y secuenciar la discusión, plantear preguntas, diagnosticar 


\begin{tabular}{lcc|c}
\multicolumn{1}{c}{ FACTORES } & $\begin{array}{c}\text { ALFA DE } \\
\text { CRONBACH }\end{array}$ & MEDIA & VARIANZA \\
\hline Factor 1: Presencia cognitiva & .902 & 3.637 & 0.137 \\
\hline Factor 2: Presencia didáctica & .871 & 3.553 & 0.036 \\
\hline Factor 3: Presencia social & .900 & 2.866 & 0.146 \\
\hline Total & .946 & 161.99 & 513.382 \\
\hline
\end{tabular}

Tabla 2. Índices de fiabilidad de las dimensiones de la escala.

errores, resumir las evidencias de aprendizaje).

3. Los indicadores para la dimensión presencia social se refieren a la expresión emocional (emoticonos, narrativas autobiográficas); la comunicación abierta (reconocimiento de los otros, expresiones de aliento); y la cohesión de grupo (alentar la colaboración, ayudar y apoyar).

Los indicadores se convirtieron en juicios, creándose un total de 36 juicios repartidos de la siguiente forma: 13 para la dimensión 1 (presencia cognitiva), 12 para la dimensión 2 (presencia didáctica) y 11 para la dimensión 3 (presencial social).

La escala fue validada mediante la revisión de jueces expertos en TIC y docencia universitaria (6 jueces), que estuvieron ampliamente de acuerdo con la pertinencia e importancia de los juicios propuestos.

Para corroborar las tres dimensiones propuestas se aplicó un Análisis Factorial mediante el método de Componentes Principales con Rotación Varimax, el cual sugiere una estructura de tres factores interpretables, que explican el $50 \%$ de la varianza. Todos los ítems, a excepción del 13 del factor 1, obtuvieron una carga superior a 0.40 en el factor relevante e inferior a 0.30 en los no relevantes, siguiendo el criterio de Hair,
Anderson, Tatham y Black (1995). Las cargas por ítem de cada factor se pueden ver en la Tabla 1. El test de Bartlett confirmó que el análisis factorial era procedente (Chi cuadrado $=6800.91 ; \mathrm{gl}=1081 ; \mathrm{p}=.000$ ).

Para analizar la fiabilidad se aplicó el coeficiente $\alpha$ de Cronbach a cada uno de los factores y al total de la escala (ver Tabla 2). Los coeficientes fueron significativamente elevados, lo que sugiere una buena consistencia interna de los constructos utilizados (Factor 1: .902 - Factor 2: .871 Factor 3: .900). Todos los factores poseen índices muy superiores a .6, mínimo aceptable según Thorndike (1997) y el total de la escala arroja un $\alpha$ de Cronbach de .946, por lo que se puede decir que la escala es muy fiable en su conjunto y con relación a cada una de las dimensiones.

\section{Resultados.}

Los resultados de tipo descriptivo mostraron por un lado la caracterización de los participantes, y por otro las percepciones del alumnado en las dimensiones cognitiva, didáctica y social del modelo teórico de Garrison, Anderson y Archer (2000). 
En cuanto a la caracterización, el reparto de estudiantes según los estudios en curso fue del $74.9 \%$ en Medicina, el $14.6 \%$ en Estudios de Maestro y el $10.5 \%$ en Educación Social. La mayoría de estudiantes se encontraban haciendo primer y segundo curso de carrera, concretamente el $60.9 \%$ hacían primer curso, el $32.1 \%$ segundo y el $7 \%$ tercer curso. Atendiendo al sexo, el $24.7 \%$ fueron hombres y el $75.3 \%$ mujeres.

En relación a la frecuencia de uso de TIC, se observó en la muestra encuestada un uso elevado de las TIC, lo que indica un alto grado de empoderamiento tecnológico. Los estudiantes manifestaron usar diferentes tecnologías diariamente o entre 2 y 4 veces a la semana. Además, las tecnologías y aplicaciones más usadas son el ordenador, el chat, el correo electrónico, los buscadores, las redes sociales y la telefonía móvil, según se puede observar en la Tabla 3.

En relación a las percepciones del alumnado sobre la presencia cognitiva, didáctica y social de su experiencia educativa con TIC, los resultados mostraron diferencias significativas entre las tres según la prueba no paramétrica de Friedman (Chi quadrado = 479.98, gl = 2, p=.000) para más de dos muestras relacionadas, una vez comprobada la no normalidad de las puntuaciones mediante Kolmogorov-Smirnov.

Como se aprecia en la Tabla 4 la presencia más destacada es la cognitiva con una media de 50.75 que considerando la puntuación teórica de la escala en este factor, supone una gran capacidad por parte del alumnado para construir significado mediante la interacción establecida en un entorno mediado por TIC. Le sigue la presencia didáctica con una media de 38.98, que indica una presencia moderada relacionada con el desarrollo de las actividades de aprendizaje y evaluación en entornos mediados por TIC.
Por último, la presencia social con una media de 28.82 se muestra como la presencia menos potenciada (muy baja si se compara con la puntuación teórica de la escala en ese factor), lo que indica la escasa capacidad de los estudiantes de proyectarse socialmente y emocionalmente en una comunidad de estudio mediada por TIC.

La observación de los descriptivos de cada uno de los ítems permitió determinar cuáles puntuaban más alto y cuáles más bajo y por tanto conocer aquellas ideas con las que estaban más de acuerdo los estudiantes (ver Tabla 5).

En general, los resultados apoyan la idea de que las TIC en la docencia son bien acogidas por el alumnado, con matices que pueden observarse en relación a cada dimensión. En la dimensión cognitiva, los estudiantes están más de acuerdo con aquellos ítems que sustentan la idea de que las TIC son una ayuda para el aprendizaje, pero no lo están con los ítems que sugieren que la TIC evitan la carga de trabajo o ayudan a aprobar las asignaturas. En la dimensión didáctica, el estudiantado está más de acuerdo con los ítems que sustentan que las TIC potencian el seguimiento de las asignaturas, pero no lo están con los ítems que apoyan un uso académico autónomo de las TIC, pues el uso solo se da en la medida que el profesorado lo sugiere. Por último en la dimensión social, el alumnado considera que las TIC (en el marco de las asignaturas) potencian los aspectos comunicativos solo con finalidad académica, pero no con finalidad personal. De hecho es la dimensión con más baja puntuación.

Para explorar posibles relaciones entre las percepciones del alumnado y las variables sociodemográficas consideradas en el estudio se aplicaron pruebas de contraste no paramétricas (por no seguir la ley normal la 


\begin{tabular}{|c|c|c|c|c|c|c|c|}
\hline $\begin{array}{c}\text { Herramientas } \\
\text { y aplicaciones } \\
\text { TIC }\end{array}$ & $\begin{array}{c}\text { No conozco } \\
\text { la } \\
\text { herramienta }\end{array}$ & Diariamente & $\begin{array}{c}\text { De } 2 \text { a } 4 \\
\text { veces por } \\
\text { semana }\end{array}$ & $\begin{array}{l}\text { Sólo los } \\
\text { fines de } \\
\text { semana }\end{array}$ & $\begin{array}{c}\text { Alguna } \\
\text { vez al } \\
\text { mes }\end{array}$ & $\begin{array}{c}\text { Alguna } \\
\text { vez al año }\end{array}$ & Nunca \\
\hline Ordenador & & 97.2 & 2.2 & 0.6 & & & \\
\hline Chat & 0.3 & 45.3 & 23.5 & 4.5 & 8.0 & 4.8 & 13.5 \\
\hline $\begin{array}{l}\text { Correo } \\
\text { electrónico }\end{array}$ & & 87.2 & 12.1 & 0.6 & & & \\
\hline Buscadores & & 75.7 & 20.4 & 1.6 & 1.9 & 0.3 & \\
\hline Redes sociales & & 62 & 18.4 & 3.5 & 4.7 & 3.2 & 8.2 \\
\hline $\begin{array}{l}\text { Páginas } \\
\text { personales }\end{array}$ & 1.6 & 9.9 & 20.5 & 6.4 & 19.2 & 7.4 & 34.9 \\
\hline $\begin{array}{l}\text { Enciclopedias, } \\
\text { diccionarios }\end{array}$ & 0.6 & 11.9 & 37.7 & 8.4 & 35.8 & 4.5 & 1.0 \\
\hline $\mathrm{P} 2 \mathrm{P}$ & 1.3 & 21.1 & 19.2 & 11.0 & 23.7 & 7.5 & 16.2 \\
\hline Lectura online & 1.0 & 17.9 & 21.1 & 11.5 & 26.2 & 8.9 & 13.4 \\
\hline Juegos online & 0.6 & 4.1 & 6.3 & 5.4 & 20.6 & 22.5 & 40.3 \\
\hline $\begin{array}{l}\text { Comercio } \\
\text { online }\end{array}$ & 1.6 & 1.0 & 1.3 & 1.6 & 21.9 & 32 & 40.5 \\
\hline Teléfono móvil & 1.0 & 84.7 & 7.0 & 1.0 & 1.3 & 1.0 & 4.1 \\
\hline MP3/MP4 & 0.6 & 52.1 & 20.8 & 1.6 & 10.1 & 2.8 & $1 ., 0$ \\
\hline Vídeo-consolas & 0.6 & 3.5 & 6.0 & 8.9 & 21.5 & 22.2 & 37.3 \\
\hline
\end{tabular}

Tabla 3. Frecuencia de uso de TIC (\%) por parte del alumnado.

\begin{tabular}{|l|c|c|c|c|c|}
\cline { 2 - 6 } \multicolumn{1}{c|}{} & N & MEDIA & $\begin{array}{c}\text { DESVIACIÓN } \\
\text { TÍPICA }\end{array}$ & MÍNIMO & MÁXIMO \\
\hline Factor1: Presencia cognitiva & 293 & 50.75 & 8.11 & 24.00 & 70.00 \\
\hline Factor2: Presencia didáctica & 291 & 38.97 & 6.02 & 20.00 & 55.00 \\
\hline Factor3: Presencia social & 289 & 28.82 & 7.35 & 10.00 & 50.00 \\
\hline
\end{tabular}

Tabla 4. Percepciones del alumnado: Puntuaciones de la escala en cada factor. 


\begin{tabular}{|c|c|c|c|}
\hline $\begin{array}{c}\text { ÍTEMS DE LAS TRES DIMENSIONES DE LA ESCALERA: } \\
\text { COGNITIVA, DIDÁCTICA, SOCIAL }\end{array}$ & $\mathbf{N}$ & MEDIA & $\begin{array}{l}\text { DESV. } \\
\text { TÍP. }\end{array}$ \\
\hline \multicolumn{4}{|l|}{ Presencia cognitiva } \\
\hline Las TIC me ayudan a adquirir conocimientos importantes en la asignatura & 310 & 4.12 & 0.693 \\
\hline Las TIC me ayudan a hacer más rápido mis tareas universitarias & 310 & 3.94 & 0.922 \\
\hline Las TIC me ayudan a hacer mejor mis tareas universitarias & 309 & 3.90 & 0.890 \\
\hline Uso las TIC para aprender & 308 & 3.92 & 0.73 \\
\hline Las TIC son mi fuente de información principal & 308 & 3.44 & 1.11 \\
\hline Considero que las TIC son importantes en mis aprendizajes & 306 & 3.95 & 0.07 \\
\hline Sin las TIC me resultaría difícil hacer mis tareas universitarias. & 309 & 3.51 & 1.06 \\
\hline Cuando quiero saber más sobre algún tema, utilizo les TIC & 308 & 3.89 & 0.92 \\
\hline Las TIC me permiten intercambiar ideas con mi profesor & 310 & 3.43 & 0.93 \\
\hline Las TIC me permiten intercambiar ideas con mis compañeros & 308 & 3.88 & 0.81 \\
\hline Las TIC me permiten asociar ideas & 310 & 3.37 & 0.82 \\
\hline Las TIC me permiten aplicar nuevas ideas & 310 & 3.45 & 0.87 \\
\hline Las TIC me descargan trabajo & 309 & 2.98 & 1.11 \\
\hline Con las TIC, aprobar la asignatura es más fácil & 310 & 2.79 & 1.00 \\
\hline Las TIC me facilitan el seguimiento de la asignatura & 307 & 3.91 & 0.77 \\
\hline Las TIC me permiten aplicar conocimiento adquirido & 307 & 3.29 & 0.87 \\
\hline Las TIC facilitan los procesos de autoevaluación & 309 & 3.72 & 0.864 \\
\hline \multicolumn{4}{|l|}{ Presencia didáctica } \\
\hline Me gusta que los profesores utilicen TIC en las asignaturas & 307 & 3.81 & 0.90 \\
\hline Las TIC facilitan fijar el programa de estudios & 306 & 3.69 & 0.83 \\
\hline Las TIC facilitan la organización de las actividades de aula & 307 & 3.81 & 0.83 \\
\hline Las TIC permiten establecer un calendario de trabajo & 304 & 3.85 & 0.85 \\
\hline Con las TIC, puedo resolver mejor mis dudas & 308 & 3.69 & 0.81 \\
\hline Con las TIC, el seguimiento de la asignatura es más cómodo & 310 & 3.86 & 0.78 \\
\hline Las TIC me permiten valorar mis avances en la asignatura & 309 & 3.35 & 0.86 \\
\hline Las TIC dinamizan las contribuciones de los compañeros & 307 & 3.53 & 0.85 \\
\hline El ritmo de trabajo se potencia con las TIC & 309 & 3.28 & 0.88 \\
\hline
\end{tabular}

Tabla 5. Percepciones del alumnado: Estadísticos descriptivos de los ítems de la escala. 


\begin{tabular}{|c|c|c|c|}
\hline El debate de los compañeros se ve potenciado con el uso de las TIC & 307 & 3.47 & 0.85 \\
\hline Las TIC me permiten evaluar mis procesos de aprendizaje & 308 & 3.41 & 0.83 \\
\hline Las TIC facilitan la presentación de los contenidos & 308 & 4.00 & 0.64 \\
\hline Las TIC me permiten confirmar lo que he entendido & 309 & 3.34 & 0.92 \\
\hline Las TIC me facilitan el diagnóstico de mis errores de aprendizaje & 308 & 3.25 & 0.86 \\
\hline Las TIC faciliten pautar la metodología del trabajo & 307 & 3.42 & 0.88 \\
\hline Las TIC me facilitan recibir ayuda del profesor & 305 & 3.58 & 0.85 \\
\hline Las TIC me facilitan la integración de conocimientos en diferentes fuentes & 305 & 3.67 & 0.78 \\
\hline Las TIC me facilitan la resolución de mis dudas & 308 & 3.71 & 0.81 \\
\hline \multicolumn{4}{|l|}{ Presencia social } \\
\hline Las TIC me facilitan una comunicación mejor con el profesor & 309 & 3.49 & 0.91 \\
\hline Las TIC me permiten una comunicación mejor con mis compañeros & 309 & 3.62 & 0.88 \\
\hline Con las TIC expreso más libremente mis emociones & 307 & 2.74 & 1.10 \\
\hline Las TIC faciliten que el profesor esté más por nosotros & 307 & 2.85 & 0.96 \\
\hline $\begin{array}{l}\text { Explico mis problemas (personales, de clase...) al profesor por medio de las } \\
\text { TIC }\end{array}$ & 307 & 2.30 & 1.06 \\
\hline Las TIC facilitan el establecimiento de un clima agradable en el aula & 307 & 2.75 & 0.96 \\
\hline Las TIC me faciliten que pueda expresarme abiertamente & 307 & 2.90 & 1.08 \\
\hline Las TIC me facilitan hacer preguntas a los otros & 301 & 3.52 & 0.90 \\
\hline Las TIC faciliten les expresiones de aprecio con el grupo & 304 & 2.81 & 1.01 \\
\hline Las TIC me permiten crear red con mis compañeros & 306 & 3.40 & 0.94 \\
\hline $\begin{array}{l}\text { Las TIC me permiten proyectar públicamente aquello que hago por las } \\
\text { asignaturas }\end{array}$ & 307 & 3.13 & 0.95 \\
\hline Las TIC me facilitan mostrarme tal y como soy & 305 & 2.41 & 1.08 \\
\hline
\end{tabular}

Tabla 5. Percepciones del alumnado: Estadísticos descriptivos de los ítems de la escala. (continuación) 


\begin{tabular}{|l|c|c|c|}
\hline \multirow{2}{*}{\multicolumn{1}{|c|}{ Prueba Kruskal-Wallis }} & \multicolumn{2}{|c|}{ ESTUDIOS QUE CURSA EL ALUMNADO } \\
\cline { 2 - 4 } & $\begin{array}{c}\text { Estudios de } \\
\text { Medicina }\end{array}$ & $\begin{array}{c}\text { Estudios de } \\
\text { Maestro }\end{array}$ & $\begin{array}{c}\text { Estudios de } \\
\text { Educación Social }\end{array}$ \\
\cline { 2 - 4 } & Media & Media & Media \\
\hline $\begin{array}{l}\text { Presencia cognitiva }(\mathrm{p}=.000 ; \mathrm{gl}=2 ; \\
\text { Chi=32.75) }\end{array}$ & 49.04 & 56.68 & 38.70 \\
\hline $\begin{array}{l}\text { Presencia didáctica }(\mathrm{p}=.000 ; \mathrm{gl}=2, \\
\text { Chi=22.16) }\end{array}$ & 38.34 & 43.07 & \\
\hline Presencia social $(\mathrm{p}=.000 ; \mathrm{gl}=2 ;$ Chi=25.71) & 27.40 & 33.38 & 30.81 \\
\hline
\end{tabular}

Tabla 6. Percepciones del alumnado según los estudios que cursa.

\begin{tabular}{|l|c|c|c|}
\hline \multirow{2}{*}{ Prueba Kruskal-Wallis } & \multicolumn{2}{l}{ CURSO EN QUE ESTÁ MATRICULADO } \\
\cline { 2 - 4 } & Primero & Segundo & Tercero \\
\cline { 2 - 4 } & Media & Media & Media \\
\hline Presencia cognitiva (p=.003; gl=2; Chi=11.34) & 49.88 & 49.72 & 57.00 \\
\hline Presencia didáctica (p=.861; gl=2, Chi=.30) & 38.92 & 38.78 & 39.45 \\
\hline Presencia social (p=.166; gl=2; Chi=3.59) & 28.09 & 29.71 & 26.65 \\
\hline
\end{tabular}

Tabla 7. Percepciones del alumnado según el curso en el que está matriculado.

distribución de datos de los tres factores). Los resultados de estas pruebas mostraron la no existencia de relaciones significativas respecto de la variable sexo y de la variable frecuencia de uso de TIC. Lo que sugiere que hombres y mujeres poseen niveles similares de la presencia cognitiva, didáctica y social de las TIC por un lado y que el hecho de usar en mayor o menor medida diferentes tipologías de TIC no afecta a la percepción de los estudiantes sobre la presencia de dichas dimensiones. En cambio sí se observaron diferencias significativas respecto a las variables estudios y curso.

En relación a los estudios la prueba Kruskal-Wallis para k muestras independientes mostró diferencias significativas en las tres dimensiones $(\mathrm{p}=.000)$, como puede verse en la Tabla 6. El alumnado de los estudios de Maestro es el que muestra una percepción superior en la presencia cognitiva, didáctica y social, seguido del alumnado de Educación Social y por último el de Medicina, como puede verse en la tabla. La diferencia por estudios permite suponer que el hecho que en las clases universitarias de los estudios del área de Educación se teorice y reflexione sobre los procesos de enseñanza y aprendizaje y sobre los procesos didácticos influye en la sensibilidad del alumnado sobre la presencia cognitiva, didáctica y social. 
En relación al curso en el que está matriculado el alumnado la prueba KruskalWallis para k muestras independientes mostró diferencias significativas en la presencia cognitiva $(\mathrm{p}=.003)$, siendo superior en el alumnado que cursa tercero de carrera, como puede verse en la Tabla 7. De todos modos, no podemos relacionar estos resultados con datos procedentes de otras investigaciones.

\section{Conclusiones.}

Los datos obtenidos en este estudio muestran cómo los estudiantes universitarios de nuestra muestra tienen acceso frecuente y generalizado a las tecnologías. Podríamos decir que son nativos digitales, de manera similar a otros estudios (Prensky, 2001, 2011). En calidad de nativos digitales, los estudiantes universitarios basan en las tecnologías de la información y la comunicación el modo de relacionarse con todo lo que les rodea, bien sea a nivel académico, lúdico o comunicativo. Del mismo modo, la valoración positiva que hacen los alumnos sobre el uso de las TIC y de las aulas virtuales es elevada, lo cual sustentaría también el perfil digital de los estudiantes universitarios. De todos modos, se hace necesario profundizar mucho más en el detalle de los usos de las tecnologías por parte de los jóvenes universitarios, para poder valorar el tipo de uso, rendimiento y provecho tecnológico.

En segundo lugar, los resultados obtenidos demuestran que los estudiantes universitarios identifican los tres tipos de presencia descritos, siendo la presencia cognitiva la más destacada, seguida de la presencia didáctica y la presencia social. A nuestro parecer, los datos aportan nuevas verificaciones a los análisis realizados en otros contextos (Shea, Li \& Pickett, 2006). En el caso investigado, una comunidad de indagación en un entorno educativo híbrido (combinando clases presenciales y campus virtual), la mayor presencia cognitiva y didáctica y la menor presencia social podría interpretarse como una respuesta al uso que hacen los estudiantes y los profesores de los foros electrónicos y los campus virtuales, que se usan de manera mayoritaria como repositorios de contenido. Ello conlleva que los campus virtuales son usados como entornos para profundizar y ampliar los contenidos, mientras que los foros electrónicos son espacios de debate. El uso comunicativo, con finalidades lúdicas, de relaciones o de socialización de los entornos virtuales, es inferior, seguramente por el hecho que los estudiantes se relacionan en las clases presenciales y dedican las sesiones virtuales al trabajo de contenidos de manera mayoritaria.

Por último, tal y como señalan Shea y Bidjerano (2009) los datos abren las puertas a nuevas investigaciones, ya que es necesario analizar no sólo la frecuencia de comunicaciones del profesor, sino también las categorías de trabajo pedagógico que incluye el diseño instruccional, la facilitación del discurso y la instrucción directa. Sin duda el diseño instructivo del aula virtual se apoya en el modelo pedagógico escogido por el docente. La distinción entre modelos de construcción guiada del conocimiento y modelos de construcción colaborativa (Mercer, 1995) podría explicar las diferencias en la presencia docente. Mientras que en el primer caso nos encontramos con una concentración máxima en el profesor, tanto del grado como de las modalidades de ejercicio de la presencia docente, en el segundo caso todos o la mayoría de participantes estarían asumiendo en un grado significativo el ejercicio de la presencia docente (Coll, Bustos \& Engel, 2011). 
Estudios orientados al contraste de ambos modelos podrían esclarecer aspectos relacionados con los resultados encontrados.

\section{Fuentes de financiación.}

Este artículo se basa en los resultados de una investigación que ha tenido una ayuda del Instituto de Ciencias de la Educación de la Universidad de Barcelona, en la convocatoria de ayudas en el Programa de Investigación en Docencia Universitaria REDICE.

\section{Referencias bibliográficas.}

Arbaugh, J.B. (2007). An empirical verication of the community of inquiry framework. Journal of Asynchronous Learning Networks, 11(1), 73-85.

Ardizzone, P. \& Rivoltella, P.C (2003). Didáctica para el e-learning. Métodos e instrumentos para la innovación universitaria. Málaga: Aljibe.

Bersin, J. (2004). The Blended Learning Book. Best Practices, Proven Methodologies and Lessons Learned. San Francisco: Pfeiffer.

Buendía, L., Colás, P. \& Hernández, F. (1998). Métodos de investigación en Psicopedagogía. Madrid: McGraw-Hill.

Coll, C., Bustos, A. \& Engel, A. (2011). Perfiles de participación y presencia docente distribuida en redes asíncronas de aprendizaje: la articulación del análisis estructural y de contenido. Revista de Educación, 354, 657-688. doi: 10.4438/1988592X-RE-2011-354-015

Fraser, J.F. (2002). Learning environments research: yesterday, today and tomorrow. En S. Goh, \& M. Khine. Studies in educational learning environments: an international perspective. (pp.1-27). Singapore: World Scientific.
Garrison, D.R. (2007). Online community of inquiry review: Social, cognitive, and teaching presence issues. Journal of Asynchronous Learning Networks, 11(1), 6172.

, Anderson, T. \& Archer, W. (2000). Critical inquiry in a text-based environment: Computer conferencing in higher education. The Internet and Higher Education, 2(2-3), 87-105.

Garrison, D.R. \& Cleveland-Innes, M. (2005). Facilitating cognitive presence in online learning: Interaction is not enough. American Journal of Distance Education, 19, 133-148. doi: 10.1207/ s15389286ajde1903_2

Hair, J., Anderson, R., Tatham, R. \& Black, W. (1995). Multivariate Data Analisis. New Jersey: Prentice-Hall.

Harasim, L. (1990). Online Education: An environment for collaboration and intellectual amplification. En L. Harasim (Ed.). Online education. Perspectives on a New Environment. (39-66). Preager: New York.

Hernndez Sampieri, R., Fernández Collado, C. \& Baptista Lucio, P. (2006). Metodología de la investigación. McGrawHill: México.

McIsaac, M.S. \& Gunawardena, C.N. (1996). Distance Education. En D.H. Jonassen Handbook on Research for Education Communications and Technology. (pp. 8-26). New York: McMillan.

Mercer, N. (1995). The guided construction of knowledge. Talk amongs teachers and learners. Clevedon: Multilingual Matters Ltfd.

New Media Consortium (2010). 2010 Horizon Report. Recuperado de http:// www.nmc.org/pdf/2010-Horizon-Reportes.pdf

Prensky, M. (2001). Digital Natives, Digital Immigrants. En el horizonte, 9 (5), 1-6. Recuperado de http://marcprensky.com/ 
writing/Prensky - Digital Natives, Digital Immigrants - Part1.pdf. doi : 10.1108/ 10748120110424816

Prensky, M. (2011). Enseñar a nativos digitales. Madrid: SM.

Kass, R.A. \& Tinsley, H.E.A. (1979). Factor analysis. Journal of Leisure Research, 11,120-138.

Rourke, L., Anderson, T., Garrison, D. R. \& Archer, W. (1999). Assessing social presence in asynchronous text-based computer conferencing. Journal of Distance Education, 14, 51-70.

Shea, P. \& Bidjerano, T (2009). Community of inquiry as a theoretical framework to foster epistemic engagement and cognitive presence in online education. Computers and Education, 52(3), 543-553. doi: doi:10.1016/ j.compedu.2008.10.007 , Li, C.S., Pickett, A. (2006). A study of teaching presence and student sense of learning community in fully online and webenhanced college courses. The Internet and Higher Education, 9, 175-190. doi: 0.1016/ j.iheduc.2006.06.005 , Swan, K. \& Pickett, A. (2005).

Developing a learning community in online asynchronous college courses: The role of teaching presence. The Journal of Asynchronous Learning Networks, 9(4), 5982.

Thorne, K. (2003). Blended Learning. How to integrate online \& traditional learning. Londres: Kogan.

Thorndike, R.M. (1997). Measurement and evaluation in psychology and education. New York: McMillan.
Fecha de recepción: $\quad$ 08-07-2013

Fecha de evaluación: 23-07-2013

Fecha de aceptación: 09-09-2013 